\title{
ON A PROBLEM OF ERDÖS CONCERNING THE ZEROS OF THE DERIVATIVES OF AN ENTIRE FUNCTION
}

\author{
K. F. BARTH AND W. J. SCHNEIDER ${ }^{1}$
}

AbStract. Let $\left\{S_{k}\right\}$ be any sequence of sets in the complex plane, each of which has no finite limit point. The authors prove, answering affirmatively a question posed by $P$. Erdös, that there exists a sequence $\left\{n_{k}\right\}$ of positive integers and a transcendental entire function $f(z)$ such that $f^{\left(n_{k}\right)^{\prime}}(z)=0$ if $z \in S_{k}$.

1. Introduction. In Hayman's function theory problem book the following problem [3, p. 17, Problem 2.30] (attributed to P. Erdös) is stated:

Let $S_{k}, k=1,2, \cdots$, be sets which have no finite limit points. Does there exist a sequence $\left\{n_{k}\right\}$ and an integral function $f(z)$ so that for every $z \in S_{k}$ we have $f^{\left(n_{k}\right)}(z)=0$ ?

The following theorem answers this question affirmatively:

THEOREM 1. Let $\left\{S_{k}\right\}$ be any sequence of sets in the complex plane, each of which has no finite limit point. Then there exists a sequence $\left\{n_{k}\right\}$ of positive integers and a transcendental entire function $f(z)$ such that $f^{\left(n_{k}\right)}(z)=0$ if $z \in S_{k}$.

For the background of this problem and related material see [1] and [2].

2. Proof of Theorem 1. We first need a lemma concerning simultaneous interpolation and approximation. The lemma is easy (but quite technical) and a direct consequence of the theorems and remarks contained in [4, pp. 310-312]. The lemma is not stated in its most concise

Presented to the Society, January 22, 1971 under the title On a problem of Erdös concerning the successive derivatives of an entire function; received by the editors December 17, 1970.

AMS 1970 subject classifications. Primary 30A66; Secondary 30A80, 30A82.

Key words and phrases. Derivatives of an entire function, interpolation in the complex plane.

1 This research was partially supported by the National Science Foundation, Grant GP20703.

(c) American Mathematical Society 1972 
form but rather in such a form that it most naturally fits into the rather complicated technical structure of the proof. For the convenience of the reader, who might not be familiar with the ideas involved, we shall briefly outline a proof in $\S 3$.

LeMma 1. Let

(1.1) $L$ be a positive integer, and let $0<r<R$,

(1.2) $A\left(=\left\{a_{1}, a_{2}, \cdots, a_{J}\right\}\right) \subset\{|z|<r\}$,

(1.3) $B_{k}\left(=\left\{b_{k, 1}, b_{k, 2}, \cdots, b_{k, m_{k}}\right\}\right) \subset\{r<|z|<R\}$ for $k=0,1, \cdots, N$,

(1.4) $\left\{\alpha_{l, k, i}: l=0,1, \cdots, L ; k=0,1, \cdots, N ; i=1,2, \cdots, m_{k}\right\}$ be any set of complex numbers satisfying the condition: If in (1.3) we have that $b_{k_{1}, i_{1}}=b_{k_{2}, i_{2}}$, then we have $\alpha_{l, k_{1}, i_{1}}=\alpha_{l, k_{2}, i_{2}}$ for $l=0,1, \cdots, L$. (This condition merely assures that the interpolation problem will be well posed when the $B_{k}$ 's are not disjoint.)

Then, given any $\varepsilon>0$, there exists a polynomial $P(z)$ such that

(2.1) $P^{(l)}\left(a_{j}\right)=0, l=0,1, \cdots, L ; j=1,2, \cdots, J$,

(2.2) $|P(z)|<\varepsilon$ for $|z|<r$,

(2.3) $P^{(l)}\left(b_{k, i}\right)=\alpha_{l, k, i}$ for $l=0,1, \cdots, L ; k=0,1, \cdots, N ; i=1$, $2, \cdots, m_{k}$.

Proof of Theorem 1. Pick $\left\{\varepsilon_{n}\right\}$ such that $\varepsilon_{n}>0$ and $\sum_{n=1}^{\infty} \varepsilon_{n}=1$. Let $f(z)$ be defined as $f(z)=\sum_{n=1}^{\infty} T_{n}(z)$, where the $T_{n}(z)$ 's are polynomials to be chosen.

To construct $T_{1}(z)$ we first pick $r_{1} \geqq 1$ such that $\left[\left\{|z|=r_{1}\right\} \cap\left(S_{1} \cup S_{2}\right)\right]=\varnothing$ and choose $n_{1}$ to be any positive integer. Then choose $T_{1}(z)$ to be any polynominal of degree greater than $n_{1}$ such that $T^{\left(n_{1}\right)}(z)=0$ for $z \in\left[S_{1} \cap\right.$ $\left.\left\{|z|<r_{1}\right\}\right]$.

Now we construct $T_{2}(z)$. We first choose $n_{2}>n_{1}$ such that $T_{1}^{\left(n_{2}\right)}(z) \equiv 0$ and then choose $r_{2}>\max \left\{2, r_{1}\right\}$ such that $\left[\left\{|z|=r_{2}\right\} \cap\left(\bigcup_{i=1}^{3} S_{i}\right)\right]=\varnothing$. Finally, we choose a point $c_{1}$ such that $r_{1}<\left|c_{1}\right|<r_{2}$ and $c_{1} \notin \bigcup_{n=1}^{\infty} S_{n}$. If, in Lemma 1, we choose:

(3.1) $\varepsilon=\varepsilon_{1}, L=n_{2}, r=r_{1}$, and $R=r_{2}$,

(3.2) $A=\left[\left(S_{1} \cup S_{2}\right) \cap\left\{|z|<r_{1}\right\}\right]$,

(3.3) $B_{0}=\left\{c_{1}\right\}$,

(3.4) $B_{1}=\left[S_{1} \cap\left\{r_{1}<|z|<r_{2}\right\}\right]$,

(3.5) $B_{2}=\left[S_{2} \cap\left\{r_{1}<|z|<r_{2}\right\}\right]$ (here, of course, $N=2$ and $m_{k}$ is the number of points in $B_{k}$ ),

(3.6) $\alpha_{l, 0,1}=\left[-T_{1}^{(l)}\left(c_{1}\right)\right]+1$ for $l=0,1, \cdots, n_{2}$ (recall that $n_{2}=L$ and $\left.c_{1}=b_{0,1}\right)$,

(3.7) $\alpha_{l, 1, i}=-T_{1}^{(l)}\left(b_{1, i}\right)$ for $l=0,1, \cdots, n_{2} ; i=1,2, \cdots, m_{1}$,

(3.8) $\alpha_{l, 2, i}=-T_{1}^{(l)}\left(b_{2, i}\right)$ for $l=0,1, \cdots, n_{2} ; i=1,2, \cdots, m_{2}$.

We see by tedious, but straightforward, checking that there exists a 
polynomial $T_{2}(z)$ satisfying:

(4.1) $T_{2}^{\left(n_{1}\right)}(z)=0$ for $z \in\left[S_{1} \cap\left\{|z|<r_{1}\right\}\right]$ (see (3.2)),

(4.2) $T_{2}^{\left(n_{2}\right)}(z)=0$ for $z \in\left[S_{2} \cap\left\{|z|<r_{1}\right\}\right]$ (see (3.2)),

(4.3) $\left|T_{2}(z)\right|<\varepsilon_{1}$ for $z \in\left\{|z|<r_{1}\right\}$ (see (3.1)),

(4.4) $T_{2}^{\left(n_{1}\right)}\left(c_{1}\right)=-T_{1}^{\left(n_{1}\right)}\left(c_{1}\right)+1$ (see (3.3) and (3.6)),

(4.5) $T_{2}^{\left(n_{1}\right)}(z)=-T_{1}^{\left(n_{1}\right)}(z)$ for $z \in\left[S_{1} \cap\left\{r_{1}<|z|<r_{2}\right\}\right]$ (see (3.4) and (3.7)),

(4.6) $T_{2}^{\left(n_{2}\right)}(z)=0$ for $z \in\left[S_{2} \cap\left\{r_{1}<|z|<r_{2}\right\}\right]$ (see (3.5) and (3.8) and note that $\left.T_{1}^{\left(n_{2}\right)}(z) \equiv 0\right)$.

It now follows that the function $\left(T_{1}+T_{2}\right)$ has the following properties:

(5.1) $\left(T_{1}+T_{2}\right)^{\left(n_{1}\right)}(z)=0$ for $z \in\left[S_{1} \cap\left\{|z|<r_{2}\right\}\right]$ (see (4.1) and (4.5) and recall how $T_{1}(z)$ was constructed),

(5.2) $\left(T_{1}+T_{2}\right)^{\left(n_{2}\right)}(z)=0$ for $z \in\left[S_{2} \cap\left\{|z|<r_{2}\right\}\right]$ (see (4.2) and (4.6) and recall that $\left.T_{1}^{\left(n_{2}\right)}(z) \equiv 0\right)$,

(5.3) $\left(T_{1}+T_{2}\right)^{\left(n_{1}\right)}\left(c_{1}\right)=1$ (see (4.4)).

(We are interpolating the values at the points $c_{i}$ in order to assure that $\sum_{n=1}^{\infty} T_{n}(z)$ is not a polynomial.)

To construct $T_{3}(z)$ we first choose $n_{3}>n_{2}$ such that $T_{2}^{\left(n_{3}\right)}(z) \equiv 0$ and choose $r_{3}$ such that $r_{3}>\max \left\{r_{2}, 3\right\}$ and $\left[\left\{|z|=r_{3}\right\} \cap\left(\bigcup_{n=1}^{4} S_{n}\right)\right]=\varnothing$. We also choose $c_{2}$ such that $r_{2}<\left|c_{2}\right|<r_{3}$ and $c_{2} \notin \bigcup_{n=1}^{\infty} S_{n}$. Making the choices analogous to (3.1)-(3.8), we can apply Lemma 1 again and obtain a polynomial $T_{3}(z)$ which satisfies

(6.1) $T_{3}^{\left(n_{i}\right)}(z)=0$ for $z \in\left[S_{i} \cap\left\{|z|<r_{2}\right\}\right]$ for $i=1,2,3$,

(6.2) $\quad T_{3}^{\left(n_{1}\right)}\left(c_{1}\right)=0$,

(6.3) $\left|T_{3}(z)\right|<\varepsilon_{2}$ for $z \in\left\{|z|<r_{2}\right\}$,

(6.4) $\quad T_{3}^{\left(n_{2}\right)}\left(c_{2}\right)=\left[-\left(T_{1}+T_{2}\right)^{\left(n_{2}\right)}\left(c_{2}\right)+1\right]$,

(6.5) $T_{3}^{\left(n_{2}\right)}(z)=-\left(T_{1}+T_{2}\right)^{\left(n_{2}\right)}(z)$ for $z \in\left[\left(S_{1} \cup S_{2}\right) \cap\left\{r_{2}<|z|<r_{3}\right\}\right]$,

(6.6) $T_{3}^{\left(n_{3}\right)}(z)=0$ for $z \in\left[S_{3} \cap\left\{r_{2}<|z|<r_{3}\right\}\right]$ (the verification here is the analog of the verification in (4.6)).

Hence it follows that the function $\left[T_{1}+T_{2}+T_{3}\right]$ has the following properties analogous to (5.1)-(5.3):

(7.1) $\left(T_{1}+T_{2}+T_{3}\right)^{\left(n_{i}\right)}(z)=0$ for $z \in\left[S_{i} \cap\left\{|z|<r_{3}\right\}\right](i=1,2,3)$ (see (6.1), (6.5), and (6.6)),

(7.2) $\left(T_{1}+T_{2}+T_{3}\right)^{\left(n_{i}\right)}\left(c_{i}\right)=1(i=1,2)$ (see (6.2) and (6.4)).

We continue inductively and define $T_{K}(z)$ in such a manner as to have the obvious analogs of (6.1)-(6.6) hold. Then we have

(8.1) $\left[\sum_{l=1}^{K} T_{l}\right]^{\left(n_{i}\right)}(z)=0$ for $z \in\left[S_{i} \cap\left\{|z|<r_{K}\right\}\right](i=1,2, \cdots, K)$,

(8.2) $\left[\sum_{l=1}^{K} T_{l}\right]^{\left(n_{i}\right)}\left(c_{i}\right)=1(i=1,2, \cdots, K-1)$, where $n_{K}, r_{K}$, and $c_{K-1}$ are defined in a similar manner to $n_{3}, r_{3}$, and $c_{2}$.

Now we set $f(z)=\sum_{K=1}^{\infty} T_{K}(z)$ and observe that $f(z)$ is an entire function by the Weierstrass $M$-test. The function $f(z)$ also has the property that $f^{\left(n_{l}\right)}(z)=0$ for $z \in S_{l}$. To see this, for any $z_{0} \in S_{l}$ choose $K_{0}$ such that $K_{0}>l$ and $r_{K_{0}}>\left|z_{0}\right|$. Then $\left[\sum_{n=1}^{K} T_{n}\right]^{\left(n_{l}\right)}\left(z_{0}\right)=0$ for $K \geqq K_{0}$ by (8.1). Hence 
$f^{\left(n_{l}\right)}\left(z_{0}\right)=0$. Of course, the possibility still remains that $f(z)$ may be a polynomial. However, this is impossible by (8.2) since there exists arbitrarily large $n_{k}$ and corresponding points $c_{k}$ such that $f^{\left(n_{k}\right)}\left(c_{k}\right)=1$, and the proof is complete.

In conclusion, we might remark that the $n_{k}$ 's depend on the $S_{k}$ 's and cannot be specified arbitrarily. For example, if we had $z_{0} \in \bigcap_{k=1}^{\infty} S_{k}$ and $n_{k}=k$ for $k=0,1,2, \cdots$, we would have $f^{(k)}\left(z_{0}\right)=0$ for $k=0,1,2, \cdots$, but $f(z) \not \equiv 0$ which is, of course, impossible.

3. Proof of Lemma 1. Pick closed disks $D_{k, i}$ about each of the $b_{k, i}$ 's with the properties:

(9.1) If $b_{k_{1}, i_{1}}=b_{k_{2}, i_{2}}$, then $D_{k_{1}, i_{1}}=D_{k_{2}, i_{2}}$.

(9.2) If $b_{k_{1}, i_{1}} \neq b_{k_{2}, i_{2}}$, then $\left[D_{k_{1}, i_{1}} \cap D_{k_{2}, i_{2}}\right]=\varnothing$.

(9.3) $\left[D_{k, i} \cap\{|z| \leqq r\}\right]=\varnothing$ and $\left[D_{k, i} \cap\{|z| \geqq R\}\right]=\varnothing$.

Let $F_{k, i}(z)$ be the polynomial of degree $L$ satisfying $F_{k, i}^{(l)}\left(b_{k, i}\right)=\alpha_{l, k, i}$ for $l=0,1, \cdots, L$. Such polynomials exist by [4, p. 49, Theorem 2]. Clearly, $F_{k_{1}, i_{1}}(z) \equiv F_{k_{2}, i_{2}}(z)$ if $b_{k_{1}, i_{1}}=b_{k_{2}, i_{2}}$ (here, of course, we are using (1.4)).

Let the function $h(z)$ be defined to be zero on $\{|z| \leqq r\}$ and equal to $F_{k, i}(z)$ on $D_{k, i}\left(k=0,1, \cdots, N ; i=1,2, \cdots, m_{k}\right)$. Then by [4, p. 47, Theorem 15], $h(z)$ can be uniformly approximated by a polynomial $\widetilde{P}(z)$ on

$$
\tilde{G}=\{|z| \leqq r\} \cup\left[\bigcup_{k=0}^{N} \bigcup_{i=1}^{m_{k}} D_{k, i}\right]
$$

Since $\widetilde{P}(z)$ uniformly approximates $h(z)$ on $\tilde{G}$, then $\tilde{P}^{(l)}(z)$ uniformly approximates $h^{(l)}(z)$ for $1 \leqq l \leqq L$ on a slightly smaller set $G$ which is the closure of an open set which contains all the points in $A \cup\left(\bigcup_{k=0}^{N} B_{k}\right)$ (for details, see [4, p. 311]). Therefore the Corollary (under the usual convention relative to multiple points) in [4, pp. 311-312] and the remarks following it apply, and hence there exists a polynomial $P(z)$ satisfying (2.1), (2.2), and (2.3).

\section{REFERENCES}

1. P. Erdös and A. Rényi, On the number of zeros of successive derivatives of analytic functions, Acta Math. Acad. Sci. Hungar. 7 (1956), 125-144. MR 18, 201.

2. - On the number of zeros of successive derivatives of entire functions of finite order, Acta Math. Acad. Sci. Hungar. 8 (1957), 223-225. MR 19, 539.

3. W. K. Hayman, Research problems in function theory, Athlone Press, London, 1967. MR 36 \#359.

4. J. L. Walsh, Interpolation and approximation by rational functions in the complex domain, 3rd. ed., Amer. Math. Soc. Colloq. Publ., vol. 20, Amer. Math. Soc., Providence, R.I., 1960. MR 36 \#1672a.

Department of Mathematics, Syracuse University, Syracuse, New York 13210 